\title{
Prediction of Volleyball Trajectory Using Skeletal Motions of Setter Player
}

\author{
Shuya Suda \\ Graduate School of Information \\ Science and Technology, \\ The University of Tokyo \\ Tokyo, Japan \\ suda@ hapis.k.u-tokyo.ac.jp
}

\author{
Yasutoshi Makino \\ Department of Complexity Science \\ and Engineering, The University of \\ Tokyo / JST PRESTO \\ Tokyo, Japan \\ yasutoshi_makino@k.u- \\ tokyo.ac.jp
}

\author{
Hiroyuki Shinoda \\ Department of Complexity Science \\ and Engineering, \\ The University of Tokyo \\ Tokyo, Japan \\ hiroyuki_shinoda@k.u-tokyo.ac.jp
}

\begin{abstract}
In this paper, we present a method that predicts the ball trajectory of a volleyball toss $0.3 \mathrm{~s}$ before the actual toss by observing the motion of the setter player. We input 3D data of body joints obtained using Kinect into a simple neural network, and 2D data estimated using OpenPose is used for comparison. We created simple neural networks for the two players and tested them. The trajectory of a volleyball toss is properly predicted by the proposed method and the error of the toss trajectory was approximately equal to the size of the ball. This technology can provide a new spectating experience in sports by superimposing the predicted images onto a live broadcast. We also show that this method can be used to identify the important body parts that contribute to the toss prediction. A professional volleyball analyst stated that this technology can be used for analyzing the peculiarities of opponent players.
\end{abstract}

\section{CCS CONCEPTS}

- Human-centered computing $\rightarrow$ Visualization

- Computer systems organization

$\rightarrow$ Embedded and cyber-physical systems

\section{KEYWORDS}

Machine learning, Ball trajectory prediction, Volleyball

\section{Introduction}

In the field of sports, data analysis and its visualization are important for understanding opponents and planning strategies. For example, in team sports such as soccer, the team strategy can be formulated by analyzing and visualizing information such as

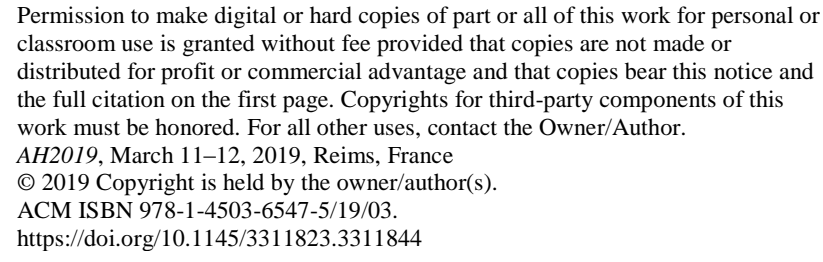

Permission to make digital or hard copies of part or all of this work for personal or classroom use is granted without fee provided that copies are not made or distributed for profit or commercial advantage and that copies bear this notice and the full citation on the first page. Copyrights for third-party components of this work must be honored. For all other uses, contact the Owner/Author. AH2019, March 11-12, 2019, Reims, France

(C) 2019 Copyright is held by the owner/author(s)

ACM ISBN 978-1-4503-6547-5/19/03

https://doi.org/10.1145/3311823.3311844

frequently existing areas and the running distance of each player. In sports wherein the movement of each player is designed for each play, such as in the case of volleyball or American football, statistical information is often used to estimate the attack pattern of the opponent team. This type of data analysis is used to capture the scene from a bird's-eye view to understand the overall strategy from a macro viewpoint.

By contrast in the case of determining the baseball pitchers' peculiarities that determines the type of pitch, the focus is on individual behaviors in the pitching motion sequence. There is a high possibility that the information that characterizes the movement is included in individual movements such as the direction of the football penalty kick, the feint in basketball, and the course of the tennis serve. In this paper, we present a method for predicting the near-future using features included in each individual body movement of the player.

We believe that there are three major applications for such shortterm predictions. One application is the superimposed display on a live video. In recent years, we can enjoy watching sports games from various angles with the development of a visual system such as VR. By superimposing the prediction images to sports video, experience of sports watching will further evolve. A schematic of this is shown in Figure 1. For example, when a soccer player attempts to dribble past a defender using the feint motion, the opponent defense player reads the movement of the attacking player and covers the area that the attacking player is expected to approach. If such information is superimposed and displayed in real time, professional bargaining can be visualized, and it is possible even for an amateur who has no experience of soccer to enjoy it. This technology can be used not only for spectators but for players. If an amateur table tennis player plays a game with AR smart glass which shows the future trajectory of the ball, the amateur player might be able to follow the ball easier. In this way, we can provide various new experiences by combining the prediction system and the recent visual technology.

The second possible application of short-term predictions is to identify a peculiarity in motion. Let us suppose that the system can predict the ball position $0.3 \mathrm{~s}$ before it is hit based on the images of the tennis serve. This means that some features that can be used for 
determining the direction of the ball are included in the sequence of the serve motion. Thus, when limiting the feature points that are to be used for learning, such as only the joints of the shoulder to the elbow, we can identify the important body part that contributes to the estimation of the ball direction. In several machine-learning methods, such as random forests, the importance of the entered feature quantities can be calculated. We believe that it is also
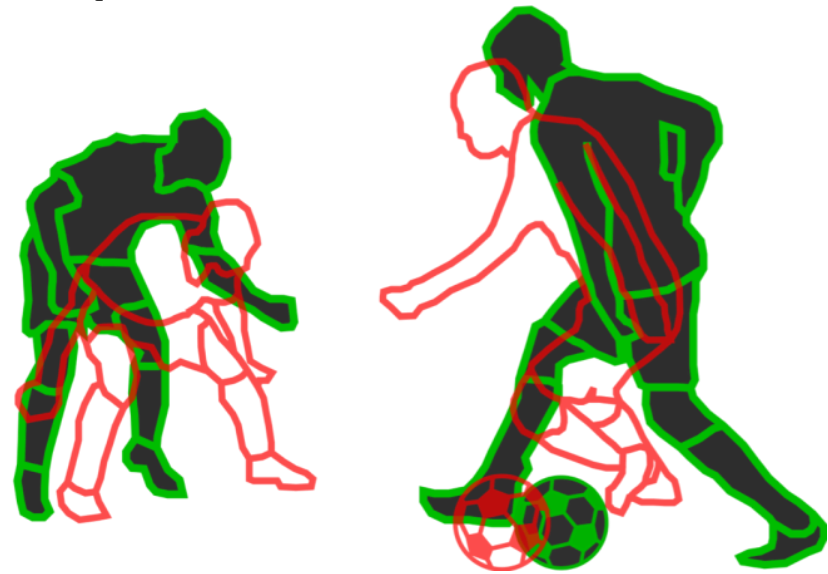

Figure 1: Soccer players are confronting each other. The current position of the players is shown in green and the future position of the players predicted by the neural network is shown in red.

possible for people to learn optimal behavior patterns that are difficult to predict by using the machine-learning results. The third possible application is to use it as a tool for technical support in sports. For example, robots that play table tennis are currently in practical use. These robots are designed to hit the ball by swinging a racket at an appropriate angle with respect to the estimated ball arrival position by using high-speed cameras and high-speed actuators. As the trajectory of a ball follows the laws of physics, a simple equation of motion and the measurement of the ball with a high-speed camera can be used to predict its position. In contrast, if it is possible to estimate the direction of the ball before the human player hits the ball based on the movement, the same is possible without using a high-speed camera or actuator.

In order to realize the above three applications, it is necessary to predict motion in real time. Therefore, it is desirable to obtain this estimation with a relatively low calculation cost. In some previous papers, they showed the method to predict human motion in realtime [5]. In this paper, we also use the similar method that uses 3D skeleton information taken by Kinect as input data for learning in a neural network to facilitate real-time performance. Compared to the previous studies, our target is not to estimate the body movement but the ball trajectory by only observing the set-up action of the volleyball setter. The system estimates how the tossed ball moves depending on the player's toss motion.

We choose the volleyball's toss motion for the estimation as our first step because of the following three reasons when considering future practical development. 1) The opponent team is separated by a net and the motion of a setter can be observed clearer than the other sports; 2) as a normal toss motion is performed at roughly same position, data acquisition is easier with a single fixed camera; and 3 ) the toss motion itself is slower than the pitching or kicking motion, and thus, sufficient motion information can be obtained using a 30 -fps camera.

In an actual game, it is difficult to measure the setter motion with a special sensor such as Kinect because the measurable range is quite limited when it is used in sports. It is necessary to perform a similar estimation using a general camera. Therefore, for comparison, we examined how the estimation accuracy changes when 2D skeleton information is provided by reducing the dimension from the $3 \mathrm{D}$ data or extracted them using OpenPose [1], which enables us to estimate human body joints from a 2D image. In the following sections, we introduce a system for estimating the trajectory of the ball and present its evaluations.

\section{Previous Studies}

\subsection{Human Motion Prediction Using Machine Learning}

Owing to recent improvements in machine learning, many complex patterns can be more easily classified or estimated than before. Many studies have been focused on forecasting the posture of the human body using a deep neural network [2] [3] [4] [5]. Based on the $3.6 \mathrm{M}$ human motion dataset [6], which comprises human skeletal data captured using a motion capture system, many types of neural networks have been proposed for modeling human actions [2] [3] [7] [8]. For example, Martinez analyzed a method of pose estimation using a recurrent neural network and proposed the new method [2]. In addition, Fragkiadaki proposed the encoderrecurrent-decoder model for the recognition and estimation of a human pose one frame before its execution [3]. In some studies, real-time human gesture recognition has been achieved using a depth map [7]. Some systems can be used to predict the trajectory of an object's motion in real-time [9] [10] [11]. For repetitive skilled motion, there exists research that discusses human motion prediction [10].

In particular, there exists research that focuses on real-time forecasting of $3 \mathrm{D}$ positions of a human skeleton $0.5 \mathrm{~s}$ before using a basic feedforward neural network. Horiuchi et al. [5] focused on the preliminary actions unconsciously included in human motion. They used Kinect and estimated the 3D coordinates of the human body parts during jumping and walking. They used 10 frames of 25 joints' position data and the center of gravity $(\mathrm{CoG})$ as a learning dataset for the neural network to predict near-future body motion. In this paper, we utilize the previously proposed neural-networkbased forecasting method [5] to predict the future trajectory of a ball instead of the motion of a body. In particular, we focus on the volleyball and body motion of a setter player.

\subsection{Forecast in Sports}

Some studies have previously been conducted on motion prediction in sports and especially tennis [12] [13] [14] [15]. For example, Shim et al. [12] reported that a human could predict the course of a tennis service based on the motion of the tennis players with a better-than-random chance irrespective of whether the player is 
skilled or a novice. In other words, it is suggested that the service motion of the tennis player potentially includes the service course, which can be perceived. Jackson et al. [13] identified which part of the tennis players' body part (or racquet) contained important information regarding the course of the tennis service. When the subjects predicted the service course, they saw only a part of the player's body, and the change in the human prediction accuracy was analyzed. Jackson et al. [13] stated that the body parts that were displayed when the prediction accuracy was high had characteristics related to the service course. Tenenbaum et al. [14] suggested that the longer the subjects watched the service motion, the more accurately they predicted the service course. Similar results are reported in [15], and it can be said that the prediction accuracy increases as the amount of obtained information regarding the player's body motion increases.

There have been many previous studies on motion prediction in not only tennis but also baseball and volleyball [16] [17] [18]. A research has been conducted to classify whether a pitch is a fastball or nonfastball with high accuracy using machine learning based on the huge pitching data of the pitcher recorded by Major League Baseball [16]. Xie et al. [17] presented a system that tracked the fast and random movements of volleyball players by constructing a model that predicted the near-future movement of their arms. In addition, Tümer et al. [18] forecasted the future ranking of the volleyball team using an artificial neural network model.

Thus, various studies have been conducted on prediction in sports. However, there is no research that predicts future ball trajectory based on the physical motion measurement of a volleyball player using machine learning. This paper differs from previous research in this respect.

\section{Methods}

Figure 2 shows the outline of the proposed system. The input of the neural network is the position of the body joints estimated using Kinect V2 and the ground truth of the ball is the 2D positions after $0.3 \mathrm{~s}$ in the image plane. The neural network learns such that the error between the output of the network and the ground truth becomes small. We explain the measurement system and prediction method in the following sections.

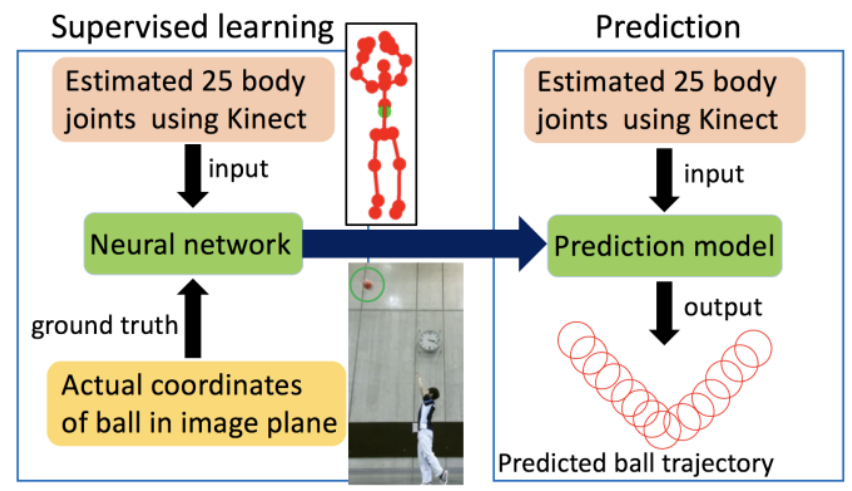

Figure 2: Outline of the proposed system

\subsection{Measurement System}

Figure 3 shows the measurement setup. We use two Kinects, which estimate the 3D positions of the body joints and ball in an orthogonal orientation, respectively. Kinect (1), which is set in front of the setter player, detects the 3D position of the joints. The ball trajectory is measured using Kinect (2), which is set up on the left side of the setter player. Although the Kinect can be used to detect depth information of the ball, we do not use the 3D information but only the 2D position. These Kinects measure the data independently at $30 \mathrm{fps}$. In order to associate the 3D positions of the body joints with the position of the ball in the image plane, we synchronize the time of each Kinect. The position of Kinects (1) and (2) are not calibrated. We use the monochrome (red) ball such that the $2 \mathrm{D}$ position of the ball can be estimated based on color tracking in the image plane.

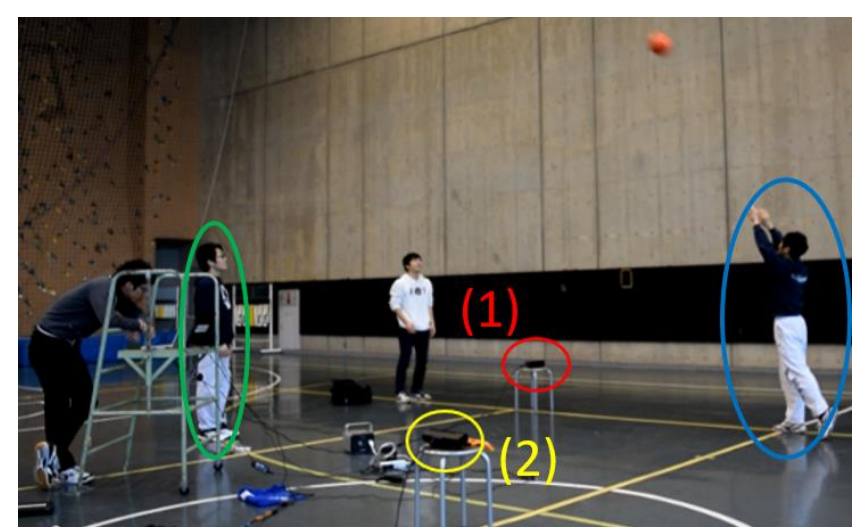

Figure 3: Measurement setup. The setter player is indicated by the blue ellipse. The person indicated by the green ellipse throws the red ball to the setter player. The setter player tosses the ball forward or backward.

\subsection{Neural Network}

In this paper, we predict the trajectory of the ball using a neural network. Figure 4 shows the neural network model we used in our system. This network has an all-connected five-layered model and a rectified linear unit as its activation function and Adam as its optimizer. In our system, we use Chainer as a machine-learning library.

We calculate the position of the CoG of the body because we considered it to be an important factor for predicting the body's motion. The $3 \mathrm{D}$ position of the $\mathrm{CoG}$ can be calculated from the $3 \mathrm{D}$ positions of each joint and the assumed mass of volume of each body segment [19]. We used the same parameters as those used in [19]. These processes are similar to those used in the previous study [5].

In order to make use of the time series patterns of the body motion, we combine the past 10 frames of the data of 25 body parts and the $z$ coordinates of the $\mathrm{CoG}$ into one learning dataset as shown in Figure 5. We continuously generate a dataset by shifting the frames 
one by one. The estimation period starts from when the thrown ball enters within the viewing angle of Kinect (2) to when the setter player touches the ball. We define this action as one toss motion.

By subtracting the position data from that of the CoG, all the coordinates of the 25 skeletons are calculated such that they are relative to the CoG. That is, the $\mathrm{CoG}$ is treated as the origin of the joint data. As a result, the time sequential pose of the setter player, which is learned using a neural network, is independent of the absolute position of the player. In addition, we input the $\mathrm{z}$ coordinates of the $\mathrm{CoG}$, which is the distance from Kinect (1) to improve the prediction accuracy of the ball motion.

The ground truth for the above input is the $2 \mathrm{D}$ coordinates of the ball 10 frames ahead of the current frame in the dataset. This neural network model learns such that it reduces the error between its output and the ground truth to a small value. The coordinates of the ball are only utilized as correct answers and not used as input for learning.

As the error function, we use the root mean squared error (RMSE), which is given as

$$
E(\boldsymbol{w})=\sqrt{\frac{1}{N} \sum_{i=1}^{N}\left\{\left(g_{i x}-e_{i x}\right)^{2}+\left(g_{i y}-e_{i y}\right)^{2}\right\}},
$$

where $\boldsymbol{w}$ is the weight vector, whose values are updated through the learning process. $g_{i x}$ and $g_{i y}$ are the $x$ and $y$ coordinate of the ground truth, respectively. $e_{i x}$ and $e_{i y}$ are the $x$ and $y$ coordinate, respectively, of the ball predicted by the neural network. $N$ represents the number of datasets.

We try to make a specialized model for an individual player since the peculiarity for predicting ball trajectory is different from person to person and we do not aim to make a universal toss-motion model that can be applied for every player. Thus, we input the skeleton of one subject per neural network model and construct the prediction model for each subject.

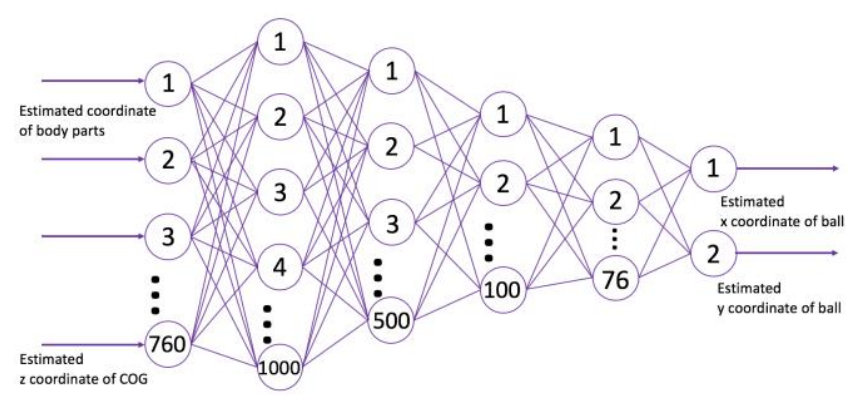

Figure 4: Neural network structure for forecasting toss trajectory.

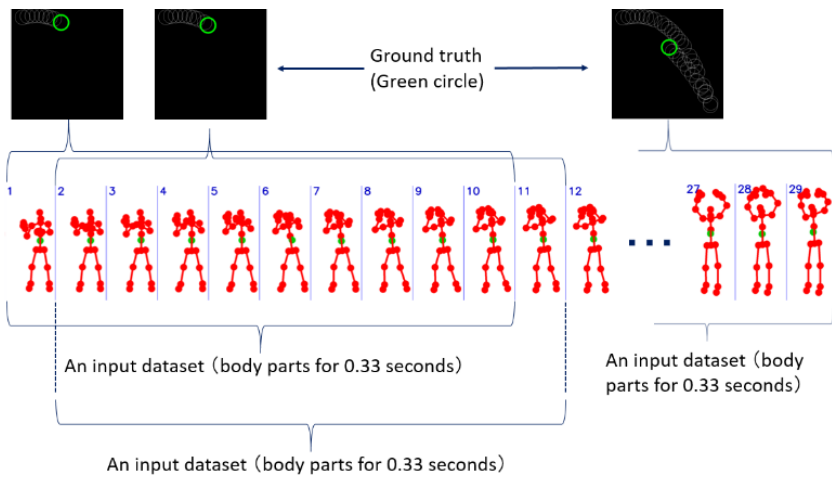

Figure 5: Input dataset to $\mathrm{NN}$ and related ground truth.

\subsection{Skeleton Extraction Using OpenPose}

In this section, we estimate the $2 \mathrm{D}$ positions of the body joints of the setter player from the 2D image of a general RGB camera using OpenPose [1], which can be used to estimate the pose of a human through machine learning from the color image and calculate the position of the joints in the image plane. If toss trajectory prediction can be achieved through body joint estimation using 2D images, there is no need to use a depth sensor at the time of measurement and it is not necessary to consider the measurement range of Kinect. In addition, there exists an advantage that the existing videos of the game can be used for analysis. As OpenPose cannot be used for commercial applications in the sports field, it cannot be used as it is for analyzing an actual game in future practical usages. In this study, we use OpenPose for verifying whether the trajectory estimation of the ball from a 2D image is possible in principle.

First of all, in order to determine which side of the body contains important information for prediction, we reduce the dimension of the $3 \mathrm{D}$ data obtained in the previous section. This means we try to imitate body joint data estimated by OpenPose by making the 3D data two-dimensional data.

Two-dimensional data is made to mimic the image taken from two different viewing positions. One condition reduces the information of $\mathrm{z}$-coordinates of the body joints. This means that the $3 \mathrm{D}$ data is reduced as if the image is taken from the front of the player, which is similar to the images shown in Figure 5. By contrast, the other one reduces the information of $\mathrm{x}$-coordinates of the body joints. This means the motion is taken from the left side of the setter player, which is similar to Figure 6.

As is shown in later section, the $2 \mathrm{D}$ body joints taken from the left side of the setter player shows higher accuracy. Therefore, we use the images of the setter player taken from his left side by using the RGB video obtained using Kinect (2). Figure 6 shows how the setter player's body joints are estimated using OpenPose. As shown in Figure 6, we can estimate the 2D positions of the body joints in the image plane even when the setter player is facing sideways. OpenPose can be used to estimate 18 body joints; however, we use 14 of them because the remaining four joints have poor estimation accuracy as they are on the hidden side.

Considering the practical use of the analysis of the existing images and predicting the trajectory of tossed balls, it is desirable to be able to use the toss motion of the setter player as an input even for images of other positions in the viewing angle. In other words, instead of the absolute coordinates observed from the camera, it is 
necessary to trim the image in the range of movement of the setter player and use the normalized coordinates within that range as the input. With this preparation, it becomes possible to input the time series pose of the setter player independent of the position of the camera.

For the normalization, we do not use the $\mathrm{CoG}$ of the body as the origin as in the case of the 3D data. The coordinates of the body joints are calculated as shown in Equation (2). This is because we assume that if we calculate the CoG position based on the body parts estimated using OpenPose, whose estimation accuracy is lower than Kinect, the error will increase, and this may cause a deterioration in the results.

$$
x_{i j}^{(N)}=\frac{x_{i j}-x_{\min }}{x_{\max }-x_{\min }}, y_{i j}^{(N)}=\frac{y_{i j}-y_{\min }}{y_{\max }-y_{\min }},
$$

Here, $x_{i j}^{(N)}, y_{i j}^{(N)}$ represents the normalized $x$ and $y$ coordinates, and $x_{i j}, y_{i j}$ represents the $x$ and $y$ coordinates before normalization. The subscript $i$ represents the position of the body joints. $x_{\max }, y_{\max }$, and $x_{\min }, y_{\min }$ are the maximum and minimum values, respectively, of the $x$ and $y$ coordinates for the whole body coordinates in one toss sequence. This means that we determine the rectangle in which the setter player moves for each toss motion whose width and height is $x_{\max }-x_{\min }$ and $y_{\max }-$ $y_{\min }$, respectively. The positions of the joints are then represented as the ratio to the width and to the height of the rectangle whose origin is set as the upper-left corner shown in Figure 7.

The coordinates normalized using Equation (2) are input into the neural network, and the toss trajectory prediction is performed in the same manner as shown in Section 3.2. We set the input layer unit to 280 instead of 760 because the number of body joints estimated using OpenPose is smaller than those estimated using Kinect and the data is $2 \mathrm{D}$. The number of units of the layer following the input layer is modified as $600,300,100,28$, and 2 in that order.

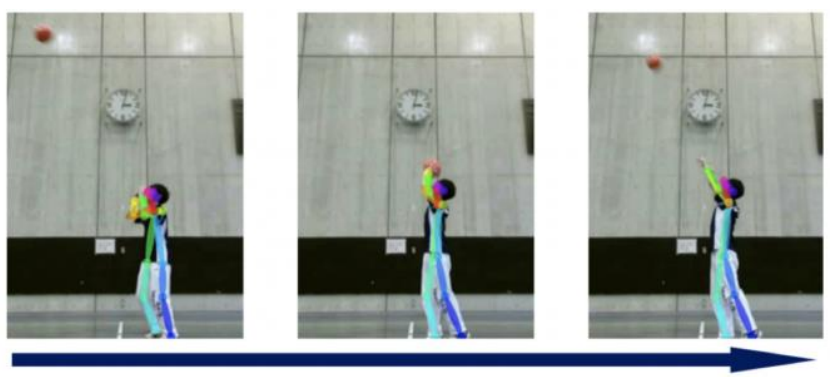

Frame

Figure 6: Body joints estimation in the image plane using OpenPose.

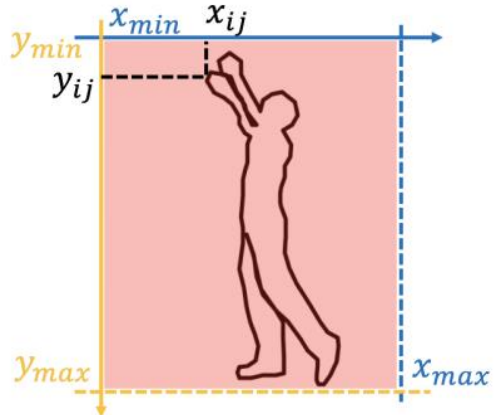

Figure 7: Position of the joints $\left(x_{i j}\right.$ and $\left.y_{i j}\right)$ are normalized. Red zone represents the range in which the setter player moves.

\subsection{Identification of Important Body Parts for Prediction}

In the previous section, all 25 joint $3 \mathrm{D}$ positions were used as the input to the neural network when the Kinect is used. In order to analyze which part of the body reflects the feature of the toss motion quantitatively, we limit the number of input joints. Figure 8 shows the image we used for learning. We change the body parts to be input and compare the obtained values of Equation (1). We chose two conditions: only arm and only lower body. We assumed that when the feature related to the trajectory of the toss is included for the input, the value of prediction error $E(w)$ will be smaller, and this means that the prediction can be performed more accurately.

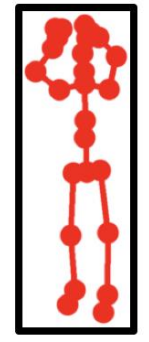

All body parts

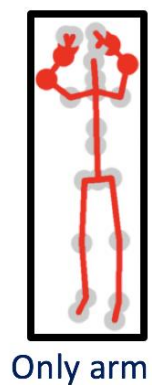

Only arm

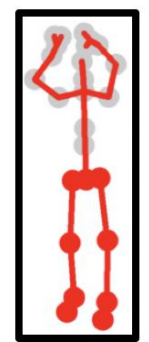

Only lower body
Figure 8: Chosen body joints input to neural network for identifying important parts.

\section{Experimental Setup}

We recorded the toss motions of two players. They have been setter players for more than 3 years. They were asked to toss the ball in two directions: forward and backward. In those cases, the ball moved in the $x-y$-plane as captured by Kinect (2) in Figure 3. In other words, the ball did not move along the $z$-axis direction of Kinect (2). The ball was always thrown to the setter player by the experimenter from the left front of the setter player. The ball was always thrown into the range where the whole body skeleton of the setter players could be observed from Kinect (1).

We measured the toss 806 times for subject 1. Among them, 703 toss motions were used as inputs for learning with the neural network. In order to evaluate the generalization performance, we 
used the remaining 103 toss data for evaluation. In the following evaluation results, the evaluation was performed using different trials from the training data.

For subject 2, we measured the toss 533 times. Among them, 464 toss motions were used as inputs for learning with the neural network. In order to evaluate the generalization performance, we used 69 toss data as evaluation data.

The toss trajectory prediction based on the 2D body joints estimated using OpenPose was performed only for subject 1 . We used 504 toss motions that could be used to estimate the body joints with a high accuracy for analysis. Among them, 454 toss motions were used for learning in the neural network. In order to evaluate the generalization performance, we used 50 toss data as evaluation data. We only analyzed the data of Subject 1 for the identification of important body parts for prediction, as mentioned in section 3.4. In each analysis, we used mini-batch machine learning. The minibatch size was 100, and the number of epoch was 100 .

\section{Results}

\subsection{Toss Trajectory Prediction}

TABLE I. Error of the prediction.

\begin{tabular}{|c|c|c|}
\hline & Subject 1 & Subject 2 \\
\hline $\begin{array}{c}\text { RMSE [cm] } \\
\text { Training Data }\end{array}$ & 20.0 & 24.4 \\
\hline $\begin{array}{c}\text { RMSE [cm] } \\
\text { Test Data }\end{array}$ & 24.7 & 29.2 \\
\hline
\end{tabular}

The learning results are shown in Table 1. As the diameter of the ball is $20 \mathrm{~cm}$, the error of the toss trajectory was approximately equal to the size of the ball in the training and test data.

Figures 9 and 10 show plots of the forecasted values and the ground truth for each frame on the image plane. Figure 9 represents the prediction of a trajectory of a backward toss for the test data for one typical trial. The red circles represent the predicted value of the ball, and the green circles represent the ground truth. From these figures, it was observed that the toss trajectory could be forecasted adequately even though the toss motion was the unlearned test data. Figure 10 shows the result of predicting of one typical trial in the test data for subject 2 for the forward toss. We confirmed that the toss direction was predicted properly in the case of both subjects. We also attempted to evaluate the result quantitatively; we evaluated how accurately the ball direction (forward or backward) is predicted using our system. That means, we treated the result as a two-class classification problem rather than a regressive prediction. We thus used 50 randomly selected tosses (25 forward and 25 backward) from both the training and test data. As a result, all the 50 tosses were appropriately predicted $(100 \%)$ in the training data. In contrast, the prediction accuracy was $96 \%$ for the test data. Here, the criteria for deciding whether or not the prediction was correct was as follows: "In the frame wherein the setter player touches the ball and its locus is changed, the direction of the ball's trajectory matches the prediction."

Based on the above results, we confirmed that the toss trajectory could be predicted properly based on 3D skeletons for two subjects.

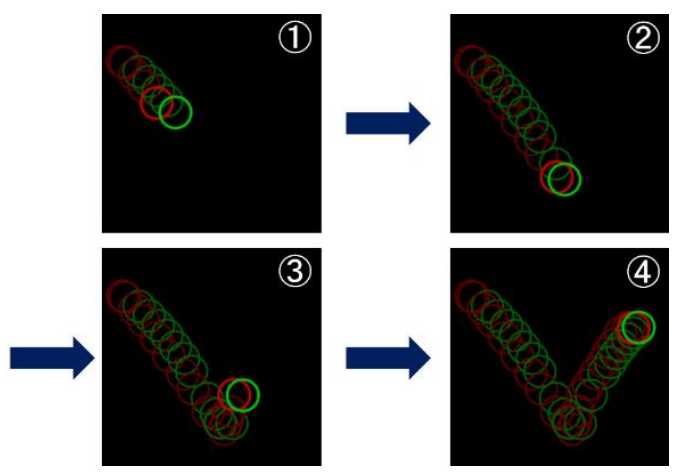

Figure 9: Prediction of toss trajectory in the backward direction of subject 1 for test data.

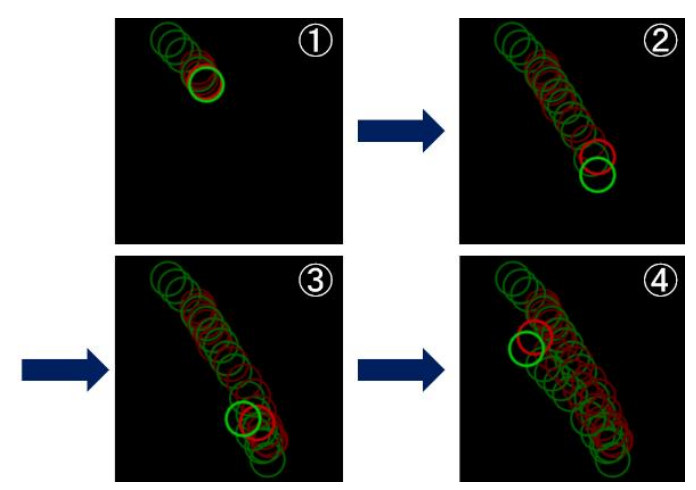

Figure 10: Prediction of a toss trajectory in the forward direction of subject 2 for test data.

\subsection{Comparison of Kinect and OpenPose}

Before estimating body joints with OpenPose, we compared the performance of the artificially generated 2D data: frontal and sideway views. As is shown in Table II, the error for 3D data and 2D sideway are comparable. By comparing the accuracy between the frontal image and the side image, the side data seems to have more information to determine the ball trajectory than the frontal image. Based on this result, we decided to use side images to estimate body joints by OpenPose.

Figure 11 show the plots of the typical forecasted values (red circles) and ground truth (green circles) for each frame on the image plane for the test data by using OpenPose. From this figure, it can be observed that the toss trajectory could be forecasted adequately even though we used 2D body joints estimated using OpenPose as the input to the neural network.

Table III shows the error of the toss trajectory prediction when we input the body joints estimated using Kinect or OpenPose into the neural network. As shown in Table III, the prediction error of the toss trajectory using OpenPose was $39.2 \mathrm{~cm}$ on average in the test data. In addition, the correct answer rate in predicting the toss direction (forward or backward from the setter) was $80 \%$ in the test data.

It was found that toss trajectory prediction can be made with a relatively high accuracy when using $2 \mathrm{D}$ body joints coordinates estimated using OpenPose, although it is inferior in accuracy from that in the case of the use of Kinect. 
TABLE II. Error Comparison with change of input dimension.

\begin{tabular}{|c|c|c|c|}
\hline & 3D & 2D-frontal & 2D-sideway \\
\hline $\begin{array}{c}\text { RMSE [cm] } \\
\text { Training Data }\end{array}$ & 20.0 & 23.1 & 20.5 \\
\hline $\begin{array}{c}\text { RMSE [cm] } \\
\text { Test Data }\end{array}$ & 24.7 & 30.3 & 23.7 \\
\hline
\end{tabular}
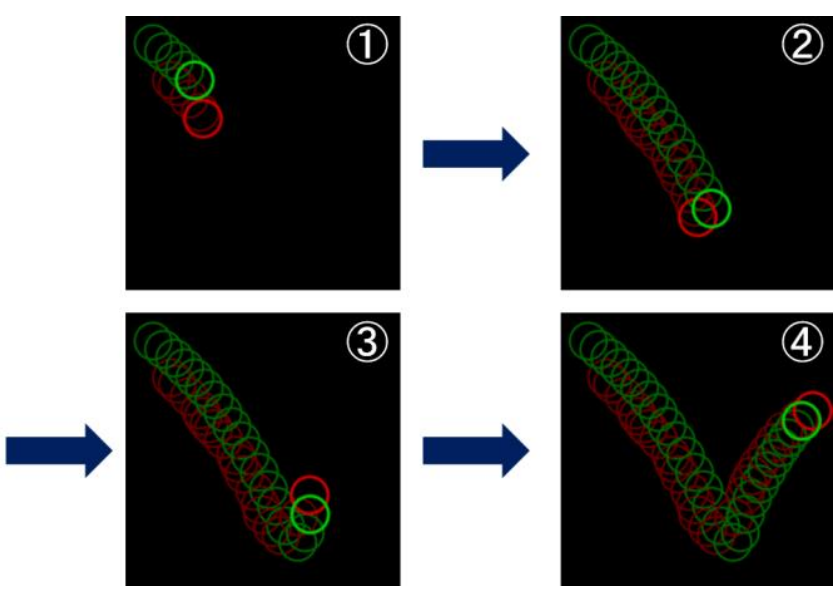

Figure 11: Prediction of toss trajectory in the backward direction of subject 1 for test data (using OpenPose).

TABLE III. Comparison of Kinect and OpenPose.

\begin{tabular}{|c|c|c|}
\hline & $\begin{array}{c}\text { Kinect } \\
\text { 25 points 3D body joints }\end{array}$ & $\begin{array}{c}\text { OpenPose } \\
\text { 14 points 2D body joints }\end{array}$ \\
\hline $\begin{array}{c}\text { RMSE [cm] } \\
\text { Training Data }\end{array}$ & 20.0 & 35.4 \\
\hline $\begin{array}{c}\text { RMSE [cm] } \\
\text { Test Data }\end{array}$ & 24.7 & 39.2 \\
\hline $\begin{array}{c}\text { Correct answer rate for direction } \\
\text { prediction for 50 toss data (test data) }\end{array}$ & $96 \%$ & $80 \%$ \\
\hline
\end{tabular}

\subsection{Identification of Important Body Parts for Prediction}

Table IV shows the comparison of the prediction error by changing the body parts that are input into the neural network. It can be observed that the prediction error is smaller in the case of the use of only the arm than that obtained on using the lower body. Although the number of joints for only the arms condition is smaller than that in the case of the lower body joints, the prediction accuracy is higher when we input the arm parts. Therefore, it is possible to quantitatively understand that the toss motion of subject 1 has influential features on the arm than those on the lower body. In addition, we focus on the plot of typical examples of the forecasted values and the ground truth in Table IV. It can be observed that the toss trajectory is qualitatively and appropriately predicted when we input the whole body parts and only the arm parts. In contrast, the figure shows that the toss trajectory is not predicted appropriately when we input lower-body parts.

We could quantitatively identify the body parts in which the motion prediction feature appears by changing the number of skeleton parts to be input.
TABLE IV. Comparison of the prediction error by changing the input body parts.

\begin{tabular}{|c|c|c|c|}
\hline & $\begin{array}{c}\text { All Body Parts } \\
\text { 25 Parts }\end{array}$ & $\begin{array}{c}\text { Only Arm } \\
\text { 6 Parts }\end{array}$ & $\begin{array}{c}\text { Only Lower Body } \\
\text { 9 parts }\end{array}$ \\
\hline $\begin{array}{c}\text { RMSE (cm) } \\
\text { Train data }\end{array}$ & 20.0 & 23.1 & 28.5 \\
\hline $\begin{array}{c}\text { RMSE (cm) } \\
\text { Test data }\end{array}$ & 24.7 & 27.7 & 35.6 \\
\hdashline $\begin{array}{l}\text { ※Subject 1 } \\
\text { ※red:estimation } \\
\text { green:real }\end{array}$ & & & \\
\hline
\end{tabular}

\subsection{Qualitative Evaluation by Professional Analyst}

In order to qualitatively evaluate the results, we asked an analyst with more than 10 years' experience at the national volleyball team to comment on our results. Here is a brief summary of the analyst's comments.

"First of all, in the field of volleyball, I have never seen the use of machine learning in predicting the ball trajectory in the near future by paying attention to body movement. I was surprised that the predicted trajectory of the ball seems considerably more accurate than I expected. However, even if you could predict the trajectory by machine $0.3 \mathrm{~s}$ before the actual motion, there is no method that can be used to tell the player about it, and thus, it is difficult to actually use this as a prediction system in a real game situation. If this technology can be used for identifying player peculiarities in advance, there is a possibility of using this for data analysis of an opponent team.

This method has to be improved in terms of following.

1) Difference between amateur and professional: There is a high possibility that many peculiarities exist in the behavior of the amateur player. As a professional player is good at eliminating peculiar motion in a toss sequence, it is necessary to verify whether a prediction is possible even in the case of the motions of a professional player.

2) Difference in data quality: The learning data of the current outcome is considerably clean. Is it possible to predict trajectories with the same accuracy when using more realistic images such as the video of a real game?

3) Data measurement method: It is practically difficult to measure the body joints using a sensor such as Kinect placed in front of a setter. Especially when it is used for analyzing an opponent team, we cannot create such a special environment. Is it possible to obtain similar estimations from camera images?"

From the comments above, the proposed method is novel in the field of volleyball, In particular, the fact that the trajectory of a ball can be estimated using such preliminary motion has not been known before. By analyzing this type of behavior, the possibility of obtaining useful information for a game in the future has been demonstrated. However, it is shown that the data set of the toss movement currently used is measured under an ideal environment, and it is necessary to use more varied data when actually using it. 


\section{Conclusion}

In this paper, we proposed a system that predicted the trajectory of the ball in the image plane $0.3 \mathrm{~s}$ in advance based on the body motion of the setter player using machine learning. We measured the motion of two subjects and predicted the trajectory of the ball in the image plane. The error was 20.0 and $24.4 \mathrm{~cm}$ in the training data and 24.7 and $29.2 \mathrm{~cm}$ in the test data for two players. We plotted the outputs of the neural network on the image plane and found that the toss trajectory could be predicted qualitatively and appropriately for both subjects.

We used OpenPose, which estimated body joints from 2D images, to compare its prediction with the prediction obtained from 3D body position data. The error was $35.4 \mathrm{~cm}$ in training data and 39.2 $\mathrm{cm}$ in the test data for subject 1 .

We also limited the inputs to the neural network to only arms or the lower body skeleton and compared each error to quantitatively detect body parts that have the feature during setting motion. As a result, it became clear that the feature of set motion was unevenly distributed amongst the body parts. This result will be applied to an application that tells the athletes about their body parts in which the characteristic motion appears. Owing to this application, the athletes can understand their individual motions and learn sophisticated forms.

\section{ACKNOWLEDGMENTS}

This research was supported by JST PRESTO 17939983.

\section{REFERENCES}

[1] Z Cao, T Simon, S-E Wei, Y Sheikh (2017). Realtime Multi-Person 2D Pose Estimation using Part Affinity Fields. In CVPR.

[2] BP Douglass. 1998. Statecarts in use: "structured analysis and object-orientation. In Lectures on Embedded Systems, Grzegorz Rozenberg and Frits W. Vaandrager (Eds.)." Lecture Notes in Computer Science, Vol. 1494. SpringerVerlag, London, 368-394. DOI:h.p://dx.doi.org/10.1007/3-540-65193-429

[3] F Katerina, et al. (2015) Recurrent network models for human dynamics. Proceedings of the IEEE International Conference on Computer Vision..

[4] J Ashesh, et al. (2016) Structural-RNN: Deep learning on spatio-temporal graphs. Proceedings of the IEEE Conference on Computer Vision and Pattern Recognition.

[5] Y Horiuchi, Y Makino, H Shinoda (2017) Computational Foresight: Forecasting Human Body Motion in Real-time for Reducing Delays in Interactive System, ISS '17 Proceedings of the 2017 ACM International Conference on Interactive Surfaces and Spaces, 312-317.

[6] I Catalin, et al. (2014) Human3. 6m: Large scale datasets and predictive methods for $3 \mathrm{~d}$ human sensing in natural environments. IEEE transactions on pattern analysis and machine intelligence $36.7,1325-1339$

[7] Chen, Chen, K Liu, and N Kehtarnavaz. (2016) Real-time human action recognition based on depth motion maps. Journal of real-time image processing $12.1,155-163$

[8] B Mohammad, et al. (2016) Neural network for dynamic human motion prediction. Expert Systems with Applications 48, 26-34.

[9] I Yuta, et al. (2016) Laplacian vision: Augmenting motion prediction via optical see-through head-mounted displays. Proceedings of the 7th Augmented Human International Conference 2016. ACM,

[10] K Hideki, and H. Yamaguchi. (2015) LumoSpheres: real-time tracking of flying objects and image projection for a volumetric display. Proceedings of the 6th Augmented Human International Conference. ACM.

[11] K Jarrod, H Benko, and AD Wilson. (2015) Juggling the effects of latency: Software approaches to minimizing latency in dynamic projector-camera systems. Adjunct Proceedings of the 28th Annual ACM Symposium on User Interface Software \& Technology. ACM

[12] J Shim, LG Carlton, JW Chow, W-S Chae (2010) The Use of Anticipatory Visual Cues by Highly Skilled Tennis Players. Journal of Motor Behavior, 164-175.

[13] RC Jackson, P Mogan. (2007) Advance visual information, awareness, and anticipation skill. Journal of Motor Behavior. 341-351.
[14] G Tenenbaum, T Sar-El, M Bar-Eli. (2000) Anticipation of ball location in low and high-skill performers: a developmental perspective. Psychology of Sport and Exercise 1, 117-128.

[15] D Farrow, B Abernethy. (2015): Can anticipatory skills be learned through implicit video based perceptual training?," Journal of Sports Science, 471-485, 2002.

[16] P Hoang, M Hamilton, J Murray, C Stafford, H Tran (2015) A Dynamic Feature Selection Based LDA Approach to Baseball Pitch Prediction. Trends and Applications in Knowledge Discovery and Data Mining, 125-137,

[17] F Xie, X Cheng, Takeshi Ikenaga (2017) Motion State Detection Based Prediction Model for Body Parts Tracking of Volleyball Players. Advances in Multimedia Information Processing - PCM 2017, 280-289.

[18] A Erdal T, S Koçer (2017) Prediction of team league's rankings in volleyball by artificial neural network method. International Journal of Performance Analysis in Sport, 202-211.

[19] P Seong-Bin, et al. (2014) A study on the development of image analysis instrument and estimation of mass, volume and center of gravity using CT image in Korean. Journal of mechanical science and technology 28.3.( 971 